\title{
détermination des coefficients élastiques d'un massif anisotrope par poinçonnement et ancrage
}

\author{
par
}

M. Dahan

P. Habib

Laboratoire de Mécanique des Solides

(E.P. - E. N. S. M. Paris - E. N.P.C. - L. A. C. N.R.S.)

M. Predeleanu

Laboratoire de Mécanique et Technologie

(Université Paris VI - E. N.S.E.T. - E.R.A. C. N.R.S.)

\section{Introduction}

Bien que le comportement rhéologique de certains dépôts de sols soit évidemment anisotrope, les difficultés d'identification des paramètres des relations de comportement ont conduit à l'utilisation de modèles isotropes beaucoup plus simples.

Dans le cas de l'élasticité linéaire, la plupart des essais in situ ont pour but de déterminer un seul paramètre d'élasticité, généralement le module d'Young, et utilisent différentes méthodes parmi lesquelles on peut citer le poinçon chargé (Habib, 1950) et le dilatomètre dans le cas des roches, ou l'essai à la plaque, l'essai pressiométrique et l'ancrage (Selvadurai-Nicholas, 1979) pour les sols.

En général, les structures stratifiées comme les sols présentent une symétrie axiale par rapport à un axe vertical, et il est raisonnable de supposer que, dans un plan horizontal, le matériau ait les mêmes caractéristiques dans n'importe quelle direction. On peut donc adopter pour ces classes de sols un modèle élastique à isotropie transverse défini par cinq coefficients d'élasticité. Pour les massifs rocheux à stratification inclinée une étude analogue peut être envisagée encore que l'hypothèse de l'isotropie dans le plan de stratification doive être considérée comme sujette à caution.

Dans tous les cas, le problème d'identification des paramètres reste difficile si l'on estime que les essais les plus appropriés pour les sols et surtout pour les roches, sont les essais in-situ. En effet, il serait nécessaire pour cela d'utiliser les solutions (nonhomogènes) de problèmes aux limites définissant les paramètres à déterminer. L'absence de ces solutions a conduit certains auteurs à se servir de solutions fondées sur une analyse isotrope pour déterminer des paramètres d'anisotropie.
Dans la présente étude, nous proposons une méthode de détermination des deux modules d'Young $E_{1}$ et $E_{2}$ $\left(E_{1}\right.$ - module d'élasticité dans la direction horizontale, $E_{2}$ - module d'élasticité dans la direction verticale) par des essais in-situ de poinçonnement et d'ancrage.

Un montage simple permet de déterminer à la fois les deux modules en admettant des relations appropriées pour les trois autres coefficients d'anisotropie.

La base théorique de cette méthode est donnée par des solutions effectives obtenues par Dahan (1979) et Dahan-Predeleanu (1981) pour le demi-espace élastique transversalement isotrope soumis à un chargement extérieur sur le plan limite (poinçonnement) et à un chargement intérieur (ancrage) couplé au précédent chargement. L'interaction des deux sollicitations permet de déduire des relations complémentaires et évite l'utilisation de tout autre système d'appui, techniquement difficile à réaliser, pour la création d'une force d'ancrage. Pour une première approche, nous avons représenté la réaction de l'ancre sur le sol par une force ponctuelle verticale.

\section{Résultats théoriques}

Nous considérons un massif semi-infini transversalement isotrope dont la surface est délimitée par le plan horizontal $(x, y)$, plan d'isotropie, et dont l'axe de symétrie élastique ( $z$ ) est vertical.

Pour un tel matériau, il est possible de définir sept constantes techniques de la façon suivante :

$E_{1}=$ module d'Young dans le plan horizontal, c'est-àdire dans le plan d'isotropie; 
$\mathrm{E}_{2}=$ module d'Young dans la direction verticale; $\nu_{1}=\varepsilon_{\mathrm{yy}} / \varepsilon_{\mathrm{xx}}$ quand la contrainte appliquée est $\sigma_{\mathrm{xx}}$; $1_{2}=\varepsilon_{\mathrm{xx}} / \varepsilon_{\mathrm{zz}}$ quand la contrainte appliquée est $\sigma_{\mathrm{zz}}$; $\nu_{3}=\varepsilon_{\mathrm{zz}} / \varepsilon_{\mathrm{xx}}$ quand la contrainte appliquée est $\sigma_{\mathrm{xx}}$; $\mu_{1}=$ module de cisaillement dans le plan horizontal; $\mu_{2}=$ module de cisaillement dans le plan vertical

Cependant, seulement cinq de ces sept constantes sont indépendantes. Nous avons en effet, deux relations supplémentaires :

$$
\begin{aligned}
& \mu_{1}=\frac{E_{1}}{2\left(1+\nu_{1}\right)}, \\
& \frac{\nu_{2}}{E_{2}}=\frac{\nu_{3}}{E_{1}},
\end{aligned}
$$

dues respectivement au plan d'isotropie $(x, y)$ et à la symétrie des coefficients élastiques.

La loi de Hooké généralisée pour de tels matériaux prend alors la forme suivante:

$$
\varepsilon_{11}=\frac{1}{\mathrm{E}_{1}} \sigma_{11}-\frac{\nu_{1}}{\mathrm{E}_{1}} \sigma_{22}-\frac{\nu_{2}}{\mathrm{E}_{2}} \sigma_{33}
$$

$$
\gamma_{23}=\frac{1}{\mu_{2}} \sigma_{23}
$$

$$
\begin{array}{ll}
\varepsilon_{22}=-\frac{\nu_{1}}{E_{1}} \sigma_{11}+\frac{1}{E_{1}} \sigma_{22}-\frac{\nu_{2}}{E_{2}} \sigma_{33}, & \gamma_{31}=\frac{1}{\mu_{2}} \sigma_{31}, \\
\varepsilon_{33}=-\frac{\nu_{3}}{E_{1}} \sigma_{11}-\frac{\nu_{3}}{E_{1}} \sigma_{22}+\frac{1}{E_{2}} \sigma_{33}, & \\
\gamma_{12}=\frac{1}{\mu_{1}} \sigma_{12} .
\end{array}
$$

\subsection{Poinçon plat à la surface du massif}

Pour le poinçonnement, sans frottement, par une fondation rigide circulaire de rayon $r_{0}$ (fig. 1), le tassement à la surface du :massif se fait suivant l'expression (Dahan-1979):

(3) $w(r)=\begin{array}{ll}\frac{P q}{4 r_{0}} & \text { pour } r \leqslant r_{0} \\ \frac{P q}{2 \pi r_{0}} \arcsin \frac{r_{0}}{r} & \text { pour } r \geqslant r_{0}\end{array}$

où $P$ est la force de poinçonnement et $q$ une constante du sol définie en fonction des coefficients élastiques par les relations suivantes:

$$
q=\frac{1+v_{1}}{E_{1}} \cdot \frac{(1-b)\left(s_{1}+s_{2}\right) \sqrt{d}}{d-a c}
$$

(5)

$$
\begin{aligned}
& s_{1}=\left[\left(a+c+\sqrt{\left.(a+c)^{2}-4 d\right)} / 2 d\right]^{1 / 2}\right. \\
& \left\{s_{2}=\left[\left(a+c-\sqrt{\left.(a+c)^{2}-4 d\right)} / 2 d\right]^{1 / 2}\right.\right. \\
& a=-v_{3}\left(1+v_{1}\right) /\left(E_{1} / E_{2}-v_{3}^{2}\right) \text {, } \\
& \mathrm{b}=\left[-v_{3}\left(-v_{3}+\mathrm{E}_{1} / \mu_{2}\right)+v_{1} \mathrm{E}_{1} / \mathrm{E}_{2}\right] /\left(\mathrm{E}_{1} / \mathrm{E}_{2}-v_{3}^{2}\right) \text {, } \\
& c=\left[-v_{3}\left(1+v_{1}\right)+E_{1} / \mu_{2}\right] /\left(E_{1} / E_{2}-v_{3}^{2}\right) \text {, } \\
& \mathrm{d}=\left(1-\nu_{1}^{2}\right) /\left(\mathrm{E}_{1} / \mathrm{E}_{2}-v_{3}^{2}\right) \text {. }
\end{aligned}
$$$$
\text { (6) }
$$

\subsection{Interaction poinçon-ancrage}

Si nous considérons l'interaction entre l'action d'une force ponctuelle $F$, appliquée à une profondeur $h$ à l'intérieur du massif anisotrope, et une fondation rigide, chargée axialement par une force $P$ (fig. 2), nous obtenons (Dahan-Predeleanu, 1981) la relation suivante entre les deux chargements et la pénétration $w_{0}$ de la fondation résultant de ces deux chargements :

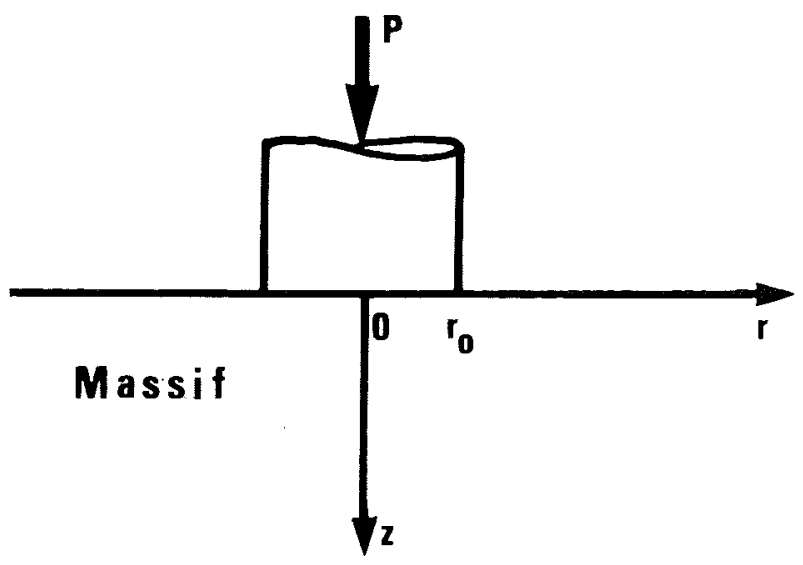

Fig. 1 Modèle de poinçon

(7) $P=\frac{4 w_{0} r_{0}}{q}-\frac{2 F}{\pi d\left(s_{1}^{2}-s_{2}^{2}\right)}$

$$
\left(g_{1} \operatorname{arctg} \frac{r_{0}}{s_{1} h}-g_{2} \operatorname{arctg} \frac{r_{0}}{s_{2} h}\right),
$$

avec les notations supplémentaires :

$$
\left\{\begin{array}{l}
g_{1}=c-d s_{1}^{2} \\
g_{2}=c-d s_{2}^{2}
\end{array}\right.
$$

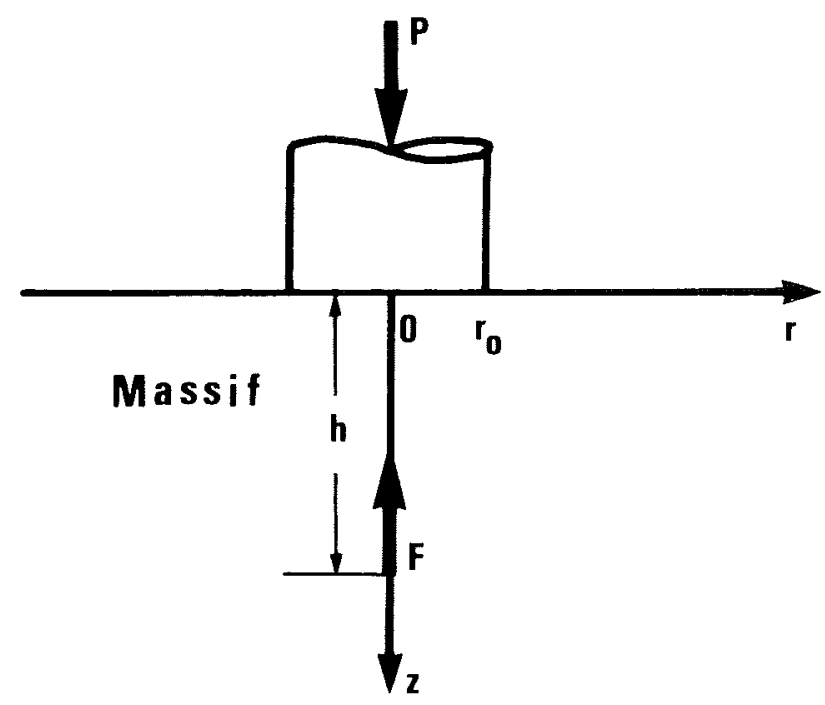

Fig. 2 Modèle de poinçon et d'ancrage

\section{Relations entre les coefficients élastiques}

- Dans les problèmes d'identification concernant les matériaux à isotropie transverse, et en particulier dans le cas des sols et des roches, il est classique d'utiliser la relation approximative de Barden (1963), obtenue à partir d'un calcul en contrainte plane, qui permet d'exprimer le module de cisaillement $\mu_{2}$ dans le plan vertical en fonction des autres coefficients. Nous admettons par conséquent la relation :

$$
\mu_{2}=\frac{E_{2} \nu_{3}}{v_{2}+v_{3}+2 v_{2} v_{3}} \text {. }
$$




\begin{tabular}{|l|l|l|}
\hline \multicolumn{1}{|c|}{$\mathrm{n}<1$} & \multicolumn{1}{|c|}{$\mathrm{n}=1$} & $\mathrm{n}>1$ \\
\hline $\mathrm{E}_{1}=\mathrm{nE}$ & $\mathrm{E}_{1}=\mathrm{E}_{2}=\mathrm{E}$ & $\mathrm{E}_{1}=\mathrm{nE}$ \\
$\mathrm{E}_{2}=\mathrm{E}$ & & $\mathrm{E}_{2}=\mathrm{E}$ \\
$\nu_{1}=v$ & $\nu_{1}=v$ \\
$\nu_{2}=v$ & $\nu_{1}=v_{2}=v_{3}=v$ & $v_{3}=v$ \\
$\nu_{3}=\mathrm{n} v$ & $\mathrm{E}$ & $\mu_{1}=\mathrm{nE} / 2(1+v)$ \\
$\mu_{1}=\mathrm{nE} / 2(1+\nu)$ & $\mu_{1}=\mu_{2}=\frac{\mathrm{E}}{2(1+v)}$ & $\mu_{2}=\mathrm{E} /(1+1 / \mathrm{n}+2 v / \mathrm{n})$ \\
$\mu_{2}=\mathrm{E} /(1+2 v+1 / \mathrm{n})$ & & \\
\hline
\end{tabular}

Les travaux d'Eftimie (1973) justifient une autre relation entre les coefficients de Poisson. Pour les matériaux tels que le rapport des modules d'Young $\mathrm{n}=$ $E_{1} / E_{2}$ soit inférieur à l'unité, il convient de poser:

$$
v_{1}=v_{2}=v \text {. }
$$

Dans le cas contraire, où $E_{1} / E_{2}$ est supérieur à un, nous avons l'égalité :

$$
v_{1}=v_{3}=\nu .
$$

Eftimie a été conduit à cette simplification en considérant la faible influence des coefficients de Poisson sur la distribution des contraintes à l'intérieur d'un massif semi-infini transversalement isotrope soumis à une charge concentrée normale à sa surface libre.

Utilisant ces nouvelles hypothèses, nous avons exprimé dans le tableau 1 les coefficients élastiques du matériau en fonction de trois constantes indépendantes $E, v$ et $n$.

Si maintenant nous introduisons ces relations dans la définition du coefficient $q$ en (4), nous obtenons trois expressions suivant les valeurs du rapport $n$ :

$$
\begin{array}{ll}
\mathrm{q}=\frac{1-\mathrm{n} v^{2}}{\sqrt{\mathrm{n}}}\left(1+\sqrt{\frac{\mathrm{n}\left(1-v^{2}\right)}{1-n v^{2}}}\right) & \text { si } \mathrm{n}<1, \\
\mathrm{q}=\frac{2\left(1-v^{2}\right)}{\mathrm{E}} & \text { si } \mathrm{n}=1, \\
\mathrm{q}=\frac{\mathrm{n}-v^{2}}{\mathrm{nE}}\left(1+\sqrt{\frac{1-v^{2}}{n-v^{2}}}\right) & \text { si } \mathrm{n}>1 .
\end{array}
$$

De même, pour le tassement à la surface du massif soumis à une charge ponctuelle intérieure, nous déduisons d'après (7) l'égalité :

$$
P=\frac{4 w_{o} r_{o}}{q}+\frac{2 F}{\pi} f\left(n, v, \frac{r_{o}}{h}\right)
$$

avec pour la fonction intermédiaire les expressions suivantes suivant la valeur du rapport des modules d'Young :

$$
\begin{aligned}
& \mathrm{f}\left(\mathrm{n}, v, \frac{\mathrm{r}_{\mathrm{o}}}{\mathrm{h}}\right)=\frac{1}{\mathrm{n}-1} \\
& {\left[n(1+v) \operatorname{arctg}\left(\sqrt{\frac{1-v^{2}}{1-n v^{2}}} \cdot \frac{r_{0}}{h}\right)-(1+n v) \operatorname{arctg} \frac{r_{o}}{\sqrt{n h}}\right]} \\
& \text { si } n<1 \\
& \text { (14) } f\left(n, v, \frac{r_{0}}{h}\right)=\operatorname{arctg} \frac{r_{0}}{h}+\frac{r_{0} / h}{2(1-v) \cdot\left(1+r_{0}^{2} / h^{2}\right)} \\
& \text { si } n=1 \\
& \mathrm{f}\left(\mathrm{n}, v, \frac{\mathrm{r}_{\mathrm{o}}}{\mathrm{h}}\right)=\frac{1}{1-\mathrm{n}} \\
& {\left[(1+v) \operatorname{arctg}\left(\sqrt{\frac{1-v^{2}}{n-v^{2}}} \cdot \frac{r_{0}}{h}\right)-(n+v) \operatorname{arctg} \frac{r_{0}}{h}\right.} \\
& \text { si } n>1 \text {. }
\end{aligned}
$$

Pour $\mathrm{n}=1$, nous retrouvons le résultat obtenu par Selvadurai (1978) pour un massif isotrope, considéré dans notre étude comme cas limite d'anisotropie. Dans les figures 3 et 4 , nous représentons les variations du coefficient $q E$ et de la fonction $f$ par rapport à la variable $n$ pour différentes valeurs du coefficient du Poisson $v$, la troisième variable $r_{0} / h$ est choisie égale à $1 / 3$. On voit sur ces figures que l'influence de $\nu$ est faible et qu'une erreur même importante sur ce coefficient n'entache pas trop la valeur de $E$.

\section{Dispositif expérimental}

Nous proposons pour l'identification des coefficients élastiques d'un massif anisotrope deux procédés de mesure. Dans ces deux essais, essentiellement statiques et pouvant s'opérer "en place", les charges appliquées au massif sont connues et les déformations résultantes sont mesurées.

Le premier est le classique essai à la plaque. Sous une plaque rigide, le sol (ou le massif rocheux stratifié) est chargé normalement et les déplacements de sa surface peuvent être exprimés en fonction de la charge totale appliquée suivant la relation (3).

Le deuxième essai est un ancrage à l'intérieur du sol, à une profondeur suffisamment grande par rapport aux dimensions de l'ancrage (6 à 8 fois) afin de supposer le chargement interne comme ponctuel. Cet ancrage est mis en interaction avec un poinçon rigide circulaire, identique à celui utilisé dans le premier essai et pouvant être représenté par la même plaque, par l'intermédiaire d'un câble. La profondeur de pénétration du poinçon vérifie alors l'équation (7) suivant les deux chargements, intérieur et extérieur (fig. 5).

Dans les essais relatifs aux terrains naturels, il est habituel de supposer connues les valeurs des coefficients de Poisson.

D'autre part, utilisant les simplifications proposées dans la section 3, les propriétés élastiques du sol ne sont alors fonction que de deux constantes $E$ et $n$ Ces dernières peuvent être déterminées à l'aide des deux procédés de mesures précédents, les relations théoriques appropriées ayant été données en (12) et (13).

Figure 5 : schéma du dispositif expérimental $w_{\mathrm{p}}=$ enfoncement de la plaque chargée à $P$. $w_{0}=$ enfoncement sous l'effet des deux chargements.

Suivant la relation (3), l'essai à la plaque permet d'obtenir la valeur du coefficient q. Reportant cette valeur dans les expressions (13) et (14), l'essai d'interaction poinçon-ancrage fournit de façon unique la valeur du rapport d'anisotropie $n$. Il est toutefois plus aisé de déduire cette valeur à partir des courbes de 

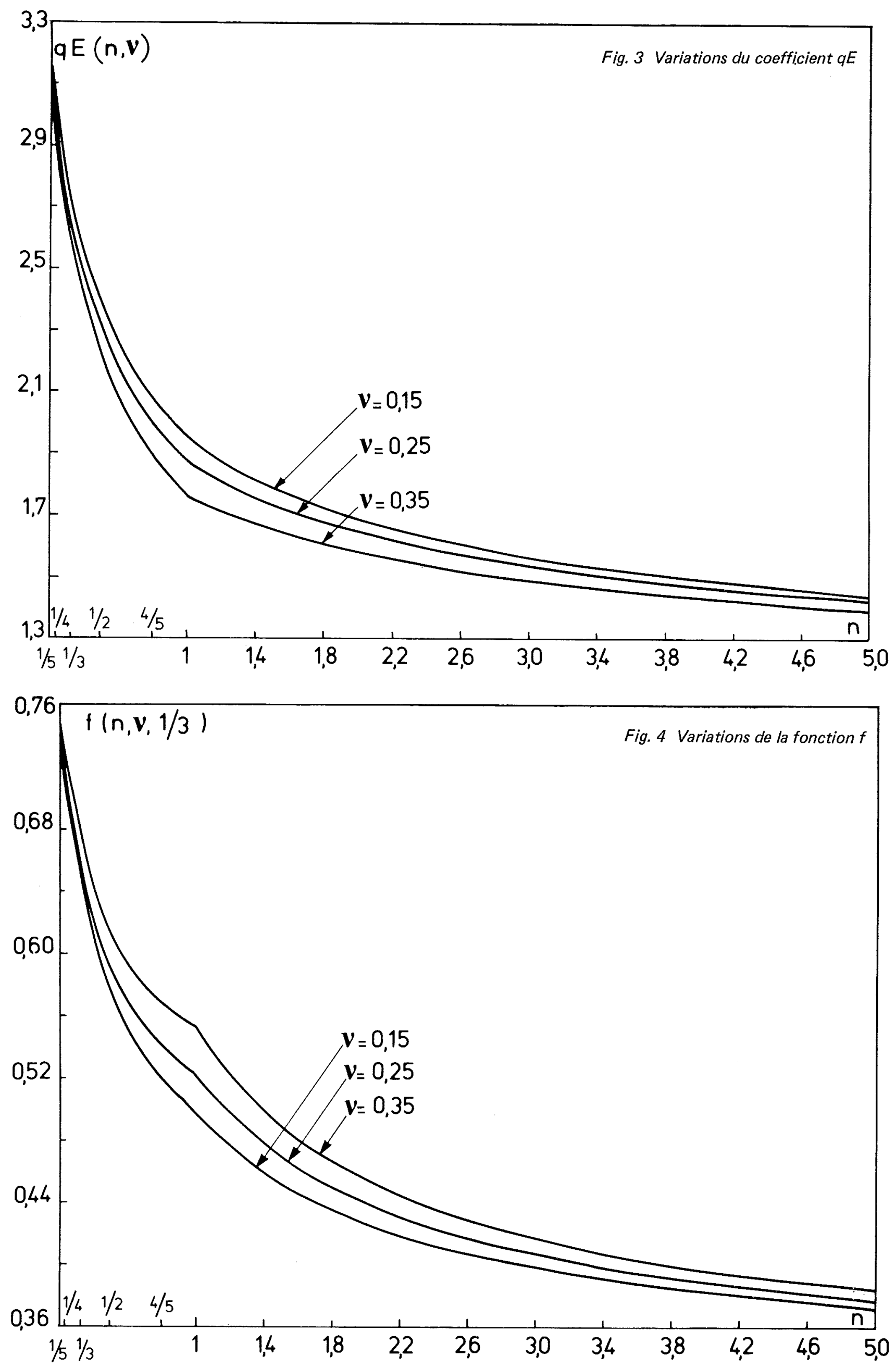
Fig. 5 Schéma du dispositif expérimental

$w_{p}=$ enfoncement de la plaque chargée à $P$

$w_{O}=$ enfoncement sous l'effet des deux chargements

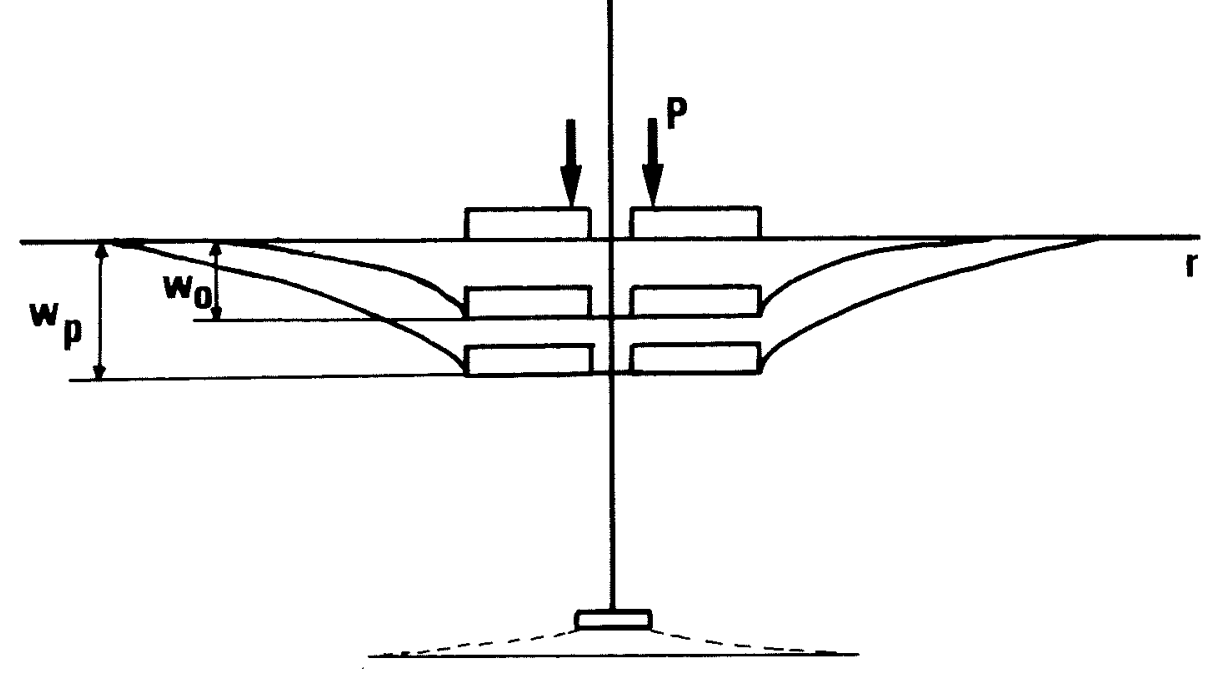

la figure 4. Connaissant $n$ et $q$, des relations (12) ou de la figure 3 , nous déduisons la valeur du module d'Young E suivant la direction verticale. Revenant au tableau 1, nous définissons les valeurs des différents coefficients élastiques du sol (ou du massif rocheux).

\section{Résultats d'essais effectués en laboratoire}

Des premiers essais sur un massif de sable sec de Fontainebleau compacté ont été effectués au laboratoire. Les dimensions du massif étaient : largeur $=$ longueur $=60 \mathrm{~cm}$, hauteur $=50 \mathrm{~cm}$. Les forces étaient appliquées statiquement par des poids éventuellement rappelés par des poulies. Les déplacements étaient mesurés par des comparateurs. Les forces de cohésion ont été simulées suivant l'application du principe des états correspondants par un vide modéré de $10 \mathrm{kPa}$. Pour un poinçon de rayon $65 \mathrm{~mm}$ et un ancrage situé à $200 \mathrm{~mm}$ de profondeur, nous avons obtenu les résultats suivants :

a) chargement sans ancrage :

Pour une charge extérieure de $\mathrm{P}=700 \mathrm{~N}$, nous avons mesuré un enfoncement du poinçon de $w=0,18 \mathrm{~mm}$. Suivant la relation (5), nous déterminons la valeur de $q$ :

$$
\mathrm{q}=\frac{4 w r_{0}}{\mathrm{P}}=6,8 \cdot 10^{-8} \mathrm{~Pa}^{-1}
$$

b) chargement avec ancrage :

Pour $P=700 \mathrm{~N}$ et une charge intérieure $F=270 \mathrm{~N}$, nous n'avons cette fois qu'un enfoncement de $0,153 \mathrm{~mm}$. D'après la relation (13) nous déduisons :

$$
f=0,607 \text {. }
$$

Prenant une valeur du coefficient de Poisson de l'ordre de 0,35 , nous obtenons à l'aide des abaques des figures 3 et 4 les valeurs suivantes:

$\mathrm{E}_{1}=18,8.10^{6} \mathrm{~Pa}=20 \mathrm{MPa}$ (module horizontal), $E_{2}=29,9 \cdot 10^{6} \mathrm{~Pa}=30 \mathrm{MPa}$ (module vertical).

Ces résultats montrent l'existence d'une certaine anisotropie, le rapport des modules d'Young $E_{1}$ étant $\overline{E_{2}}$

égal à 0,66 . On constate que ce massif de sable compacté paraît plus raide dans le sens vertical que dans le sens horizontal, ce qui paraît normal pour une mise en place par déversement suivi d'un compactage dans la direction verticale.

\section{Conclusion}

La méthode présentée dans ce texte de détermination des deux modules d'Young par l'intermédiaire de poinçon permet de mieux connaître le comportement global d'un corps anisotrope. Pour des massifs pas très homogènes, elle donne probablement des résultats plus réalistes que les essais classiques de mesures des coefficients élastiques à partir d'éprouvettes prélevées ponctuellement dans différentes directions du matériau.

Pour des essais en place avec des plaques rigides en appliquant les relations générales données en (3) et (7) entre les forces appliquées et les déplacements résultants, on obtient aisément à l'aide des abaques présentés les valeurs des modules d'Young et le degré d'anisotropie du massif considéré.

\section{Références bibliographiques}

BARDEN L. (1963) - Stresses and displacements in a Cross-anisotropic Soil. Géotechnique 13, 198-210.

DAHAN M. (1979) - Poinçons axisymétriques rigides sur un massif élastique semi-infini transversalement isotrope. Journal de Mécanique Appliquée, 3, 373-386.

DAHAN M., PREDELEANU M. (1981) - Interaction between internal loading and surface indentation for a transversely isotropic elastic half-space. Acta Mechanica (sous presse).

EFTIMIE A. L. (1973) - Starea de tensiune in terenurile anisotrofe de fundatie. Buletinul Inst. Politechnic din lasi, Sectia V, XIX, 23-29.

HABIB P. (1950) - Détermination du module d'élasticité des roches en place. Annales ITBTP, 145, 3, 2735.

SELVADURAI A.P.S. (1978) - The interaction between a rigid circular punch on an elastic halfspace and a Mindlin force. Mech. Res. Comm., 5, 5764.

SELVADURAI A.P.S., NICHOLAS T. J. (1979) - A theoritical assesment of the screw plate test. 3rd Int. Conf. Num. Methods in Geomechs, Aachen, 2-7 avril $1979,1245-1252$. 
